\title{
Clinical Findings and Treatments of 21 Children with COVID-19: A Comparison with Their Families
}

\author{
Herui $\mathrm{Li}^{1}$, Yiming $\mathrm{Ma}^{1}$, Zihang Zeng ${ }^{1}$, Huihui Zeng ${ }^{1}$, Zhiguo Zhou ${ }^{2}$, Qimi Liu ${ }^{3}$, Peng \\ Huang ${ }^{4}$, Wenlong Liu ${ }^{5}$, Mingyan Jiang ${ }^{6}$, Ping Chen ${ }^{1}$, and Yan Chen ${ }^{1}$ \\ ${ }^{1}$ Second Xiangya Hospital \\ ${ }^{2}$ First Hospital of Changsha \\ ${ }^{3}$ The second people's hospital of Guilin \\ 4Zhuzhou Central Hospital \\ ${ }^{5}$ Yueyang Second People's Hospital \\ ${ }^{6}$ Xiangtan Central Hospital
}

May 27, 2020

\begin{abstract}
Objective: To deepen the understanding of clinical characteristics of pediatric patients with COVID-19. Methods: Retrospective analysis was executed among 21 children and their 38 families with laboratory-conformed novel coronavirus disease in five designated hospitals. Results: In total of 21 children (10 males and 11 females) aged from 1 to 17 years, 90.5\% were from 18 family clusters and admitted to designated hospitals 2 days after illness onset, shorter than the adults. Besides 5 asymptomatic cases, pediatric patients mainly performed as fever (66.7\%), cough (19.0\%) and expectoration (14.3\%). Among the 38 adult patients, the major symptoms included fever (63.2\%), cough $(63.2 \%)$, expectoration (44.7\%), fatigue (6.8\%), chill (15.8\%) and dizziness $(15.8 \%)$. Most cases had normal $(76.2 \%, 68.4 \%)$ or decreased $(19.0 \%, 26.3 \%)$ leucocytes, nearly half of children showed decreased neutrophils. 15 children and 32 adults had pulmonary involvement, consisted of ground-glass opacity (57.1\%, $60.5 \%)$, spots $(14.3 \%, 23.7 \%)$, stripes $(14.3 \%, 7.9 \%)$ and patchy shadow $(9.5 \%, 36.8 \%)$. All adults received antiviral therapy: lopinavir/ritonavir (92.1\%), interferon (76.3\%), arbidol (71.1\%), chloroquine (18.4\%) and ribavirin (10.5\%). 16 children used interferon; 12 took lopinavir/ritonavir; 7 were given arbidol; two received ribavirin. All patients had been recovered and discharged with duration of admission 4-47 days (median 17 days). Conclusions: Pediatric patients with COVID-19 presented as milder symptoms and limited lung lesions than adults. Laboratory abnormalities were atypical in mild patients, neutropenia may be a potential characteristic of children. Efficiency of antiviral therapy need to be further investigated.
\end{abstract}

\section{Introduction}

Since coronavirus disease 2019 (COVID-19) outbroke in Wuhan, China, more than 5,404,512 cases confirmed and 343,514 deaths had been reported worldwide up to $26^{\text {th }}$ May $2020^{1}$. At the beginning of the epidemic, children were thought less susceptible to severe acute respiratory syndrome coronavirus 2 (SARS-CoV-2) in consequence of few patients reported ${ }^{2}$. Then, pediatric patients gradually increased with the deeper cognition of family clustering ${ }^{3}$. A large case series by the Chinese Center for Disease Control and Prevention ${ }^{4}$ included 72,314 patients in China mainland, among which less than $1 \%$ cases were younger than 10 years old, and $1.23 \%$ cases ranged from 10 to 19 years old. Although clinicians reported the clinical and epidemical features in some retrospective studies, understanding of pediatric patients with COVID-19 was still limited due to small sample size and incomplete information. Herein, we summarized and compared clinical processes of children and their families with COVID-19, in order to provide valuable experiences and give some insights.

Materials and Methods 
Study design and Participants

21 children and their 38 families were included from a series of 444 inpatients confirmed COVID-19 by the presence of SARS-CoV-2 in nasopharyngeal swab specimens detected by real-time reverse transcriptionpolymerase chain reaction (RT-PCR) assay in five designated hospitals from 21st January 23rd to March 23. In accordance to the Chinese prevention and control protocol for COVID-19 (6th edition), all patients were further assessed and classified into mild and severe cases. Then, we collected and analyzed clinical information of them.

The study protocol was approved by the institutional ethics committee of the First Hospital of Changsha, the Second Xiangya Hospital of Central South University, Zhuzhou Central Hospital, Xiangtan Central Hospital, Yueyang Second People's Hospital and People's Hospital of Yunyang District in Shiyan City, informed consent was waived because of the retrospective design.

Data acquisition

The data of all patients were provided by the Public Health Treatment Center of Changsha, Zhuzhou Central Hospital, Xiangtan Central Hospital, Designated Hospital of Junshan District, Yueyang, Hunan and People's Hospital of Yunyang District in Shiyan City, Hubei. Demographics, epidemics, clinical symptoms and signs, history of exposure, comorbidities, laboratory tests, chest computed tomography (CT) scan images, treatment and clinical outcomes were collected from the medical records without intervention.

The date of illness onset was defined as the day when the patients initially became symptomatic or the asymptomatic cases had a positive result of RT-PCR test for SARS-CoV-2. All patients underwent CT scan in the course of COVID-19. We summarized image findings according to the morphology of lesions, which were described as ground-glass opacity (GGO), spots, stripes and patchy shadow. Pleural effusion were assessed as accompanying signs.

\section{Definitions}

Clearance of virus was defined as having at least two consecutive negative results for nasopharyngeal swab specimens collected 24 hours apart. The length of virus clearance was calculated from the first date of positive RT-PCR to that negative.

\section{Statistical Analysis}

All information was entered and verified by three researchers independently, with SPSS 18.0 software (SPSS Inc., Chicago, IL, USA) for the data processing. Data were presented as median (interquartile range, IQR) for continuous variables, count (percentage) for categorical variables. Comparisons between children and adults were analyzed using student t or Mann-Whitney's tests for continuous arguments, chi-square or Fisher's exact tests for categorical parameters.

\section{Result}

Demographic and Clinical Information

Our retrospective analysis identified 444 patients with laboratory-proven novel coronavirus pneumonia at all ages. 21 cases under 18 years old were included, with 10 boys and 11 girls. The patients ranged from 1 to 17 years of age (median 8 years; IQR 3-13.5 years). 10 patients (47.6\%) had a specific history of exposure to Hubei, including residence in Wuhan (three cases), Wuhan or Hubei tourism (three cases) and close contact with people who had returned from Wuhan (four cases). A total of $90.5 \%$ patients were from 18 family clusters, which had an average of 3.47 confirmed members (ranged from 2-7 patients). Thus, their 38 families (21 male and 17 female), from 18 to 84 years of age, were collected and made a comparison. Duration from illness onset to admission ranged from 1 to 7 days (median 2 days; IQR 1-3 days) in children, which were significantly shorter than adults (median 4 days; IQR 2-7 days). Table 1 summarized the demographic and clinical data. 
Besides a boy diagnosed congenital malformation of infantile urinary system during hospitalization, $95.2 \%$ children had no identified underlying disease or predisposing condition, which were more common in adults (44.70\%). Among 17 adults with comorbidities, hypertension (11, 64.7\%), diabetes mellitus (4, 23.5\%), cardiovascular diseases $(3,17.6 \%)$, rheumatic diseases $(3,17.6 \%)$ and respiratory disease $(2,11.8 \%)$ were the most common.

All pediatric subjects presented as mild pneumonia, five (23.8\%) were asymptomatic and detected by positive results of RT-PCR test for SARS-CoV-2 in the screening people. Among $76.2 \%$ children had clinical manifestations documented, fever $(14,66.7 \%)$, cough $(4,19.0 \%)$ and expectoration $(3,14.3 \%)$ were the most common symptoms. Convulsion, projectile vomiting, delirium and opisthotonos were observed in a 2-year-old girl after fever of 39.1 without meningeal irritation signs, which were identified as febrile convulsion. Clinical manifestations were more frequent and variety in adults: fever $(24,63.2 \%)$, cough $(24,63.2 \%)$, expectoration $(17,44.7 \%)$ and fatigue $(14,36.8 \%)$.

\section{Laboratory Examination}

A summary of laboratory findings was shown in Table 2. $95.2 \%$ pediatric cases had normal or decreased leucocytes counts. 10 children showed neutropenia, which more common than their families. Abnormalities of lymphocytes and blood biochemistry were rare, but increased creatinine was observed in $63.2 \%$ adults. As for inflammation indicators, increased erythrocyte sedimentation rate, procalcitonin, C-reactive protein were observed in $47.6 \%, 38.1 \%, 4.8 \%$ of children and in $76.3 \%, 23.7 \%, 42.1 \%$ of adults.

\section{Radiological Findings}

Table 3 summarizes the radiological characteristics of patients. $28.6 \%$ children and $15.8 \%$ adults had no clear evidence of pneumonia in CT images. The most common lesions were ground-glass opacity, which were observed in asymptomatic cases. Compared with the children, adults often showed bilateral involvement with patchy infiltration.

Treatment and Outcomes

Table 4 showed the treatment and outcomes in the 21 children and 38 adults.

Among 19 pediatric patients received antiviral therapy, 16 accepted interferon inhalation (12 cases) or nasal spray (4 cases); 12 took oral lopinavir/ritonavir; 7 took oral arbidol; 2 received ribavirin intravenous drip. The patient who combined with central nervous system involvement was treated with intravenous methylprednisolone, budesonide inhalation and intravenous immune globulin. Differently, adults received more respiratory supports and antiviral therapy: using oral lopinavir/ritonavir, inhaled interferon and oral arbidol the most; the use of lopinavir/ritonavir, arbidol and chloroquine were more common than that in children.

Most subjects had no identified complications, one child was detected liver dysfunction during hospitalization, of whom the antiviral treatment were lopinavir/ritonavir and ribavirin. All individuals had been recovered and discharged after confirmation of virus clearance. The length of virus clearance and admission duration were 15 and 17.0 days in children, 14.0 and 16.5 days in adults.

\section{Discussion}

Severe acute respiratory syndrome coronavirus 2 (SARS-CoV-2), first reported in Wuhan of Hubei province, China, causes respiratory and systemic illness with poor clinical outcomes associated with older age and underlying health conditions ${ }^{5}$, continues to progress toward global pandemic. Children was paid less attention in consequence of rare subjects, perpetuating a knowledge deficit of pediatric health care. This study showed a series of minors with novel coronavirus infection, made comparisons with their families, aimed to deepen understanding of pediatric infectors and make insights.

In total of 444 laboratory confirmed inpatients at all ages, pediatric individuals accounted for 4.7 percentage. Lee et $\mathrm{al}^{6}$ inferred that children have fewer outdoor activities and international travel, making them less likely 
to contract the virus initially. Besides, coronavirus was reported involved respiratory tract by angiotensinconverting enzyme 2 (ACE2) as the cell receptor in humans ${ }^{7}$, which was thought to be purported mechanisms of epidemical characteristics in children. In our cohort, all cases had specific history of contact or exposure, together with closer distance to epidemic area, led to higher percentage of children than the data from the Chinese Center for Disease Control and Prevention ${ }^{4}$. It also confirmed that familiar transmission was the main route of pediatric infectors with novel coronavirus ${ }^{3}$. Therefore, the importance of personal protection should be further figured out to prevent COVID-19.

Our clinical findings suggested that pediatric patients were admitted to hospital 1-7 days after symptom onset, significantly shorter than adults ${ }^{3,5}$, had mild manifestations ${ }^{8}$. Asymptomatic individuals, which were detected by nucleic acid test after their family members were diagnosed, became potential source of infection ${ }^{9}$. Fever was the most common symptoms in children and their families, followed by cough and expectoration: more frequent in adults. Since older age and comorbidities were risk factors for severity ${ }^{5}$, adults had more symptoms.

No consistent derangements were observed in laboratory indices. A normal leukocyte count was found in most cases relative to the locally defined reference limits. No lymphopenia was found in children, but $13.2 \%$ adults did. Nearly half $(47.6 \%)$ of children showed neutropenia-a 17 -year-old boy presented as abnormal blood routine with leukocyte count of $2.31\left(\times 10^{9} / \mathrm{L}\right)$ and neutrophils count of $0.63\left(\times 10^{9} / \mathrm{L}\right)$, had longest duration of viral shedding (39 days). On the contrary, a review of laboratory abnormalities reported increased neutrophils and decreased lymphocytes as main characteristics of the included studies ${ }^{5}$. The foregoing differences reflected atypical changes of laboratory indices in mild cases, which need large samples to reach a consensus. We summarized three inflammatory biomarkers: erythrocyte sedimentation rate, procalcitonin and c-reactive protein, with the rates of abnormality $47.9 \%, 38.1 \%, 4.8 \%$ in children and $76.3 \%, 23.7 \%, 42.1 \%$ in adult, indicated that erythrocyte sedimentation rate was more sensitive at an early stage of novel coronavirus infection.

Chest computed tomography scanning was an important diagnostic tool of screening infectors with negative pathogen identification ${ }^{11}$. Radiology of novel pneumonia mostly presented as ground-glass opacity in unilateral or bilateral lung, variable lesions also were observed ${ }^{12}$. In our study, $57.1 \%$ children and $60.5 \%$ adults had ground-glass opacity: including symptomatic and asymptomatic infectors. Spots, stripes and patchy shadow were found rarely in children and commonly in the symptomatic or adult individuals, indicated severe illness ${ }^{13}$. Pleural effusion, which was thought more common at the stage of the disease progressed ${ }^{14}$, involved one adult. More significantly, the absorption of pulmonary lesions lagged behind the improvement of clinical manifestations and nucleic acid detection ${ }^{15}$.

The management modalities of COVID-19 infection consist of antiviral therapy, symptomatic and respiratory supporting. Antibiotic therapy, glucocorticoid and intravenous immunoglobulin therapy were also considered base on the patients' conditions. Early antiviral treatment contributed to alleviate the severity and improve the prognosis of patients with COVID-19 ${ }^{16}$. In this study, patients were mainly treated by interferon, lopinavir/ritonavir, arbidol, ribavirin and chloroquine, had a better prognosis $(100 \% \text { recovery })^{17}$; lopinavir/ritonavir, arbidol and chloroquine were used more in adult. Although all of the above drugs were recommended medicine by WHO and National Health Commission of the People's Republic of China, interferon sprays and aerosol inhalation was only recommended in children owing to its efficiency of reducing symptoms and viral load ${ }^{18}$. Moreover, safety and efficiency should be considered carefully before using in pediatric patients.

There are several limitations in the current study deserved to be acknowledged. First, the sample size was small. Second, the cases aged from 1 to 17, with different status of their conditions, may lead to some deficiencies. Third, the natural limitations of retrospective analysis may introduce the possibility of unrecognized biases.

\section{Conclusion}

In summary, familiar contact was the main route of transmission outside of Wuhan. Pediatric infectors of 
SARS-CoV-2 presented as milder symptoms and limited pulmonary lesions than their families. Ground-glass opacity was the most common lesion, pleomorphic lesions may indicate severe and progressed disease. We found atypical neutropenia in nearly half of children, indicated different peripheral blood changes. Furthermore, larger sample studies should be designed to investigate abnormalities of lab and antiviral treatment, aimed to reach on the consensus.

\section{Acknowledgments}

Author contribution: Dr. Y. Chen conduct and supervised this study. All data of patients were collected by Dr. Y. Ma, Dr. Z. Zeng, Dr. H. Zeng, Dr. Z. Zhou, Dr. Q. Liu, Dr. P. Huang, Dr. W. Liu, Dr. M. Jiang and Dr. P. Chen. Dr. H. Li summarized the data, drafted the manuscript and undertook the systematic review and modifying with Dr. Y. Ma and Dr. Y. Chen. In addition, we appreciate medical workers of five designated hospitals in the current study.

\section{References:}

1. World Health Organization, Coronavirus disease 2019 (COVID-19): situation report- 127.

2. Huang C, Wang Y, Li X, Ren L, Zhao J, Hu Y, Zhang L, Fan G, Xu J, Gu X, Cheng Z, Yu T, Xia J, Wei Y, Wu W, Xie X, Yin W, Li H, Liu M, Xiao Y, Gao H, Guo L, Xie J, Wang G, Jiang R, Gao Z, Jin Q, Wang J, Cao B. Clinical features of patients infected with 2019 novel coronavirus in Wuhan, China. The Lancet 2020;395(10223):497-506.

3. Chan JF, Yuan S, Kok K, To KK, Chu H, Yang J, Xing F, Liu J, Yip CC, Poon RW, Tsoi H, Lo SK, Chan K, Poon VK, Chan W, Ip JD, Cai J, Cheng VC, Chen H, Hui CK, Yuen K. A familial cluster of pneumonia associated with the 2019 novel coronavirus indicating person-to-person transmission: a study of a family cluster. The Lancet 2020;395(10223):514-523.

4. Z W, JM M. Characteristics of and Important Lessons From the Coronavirus Disease 2019 (COVID-19) Outbreak in China: Summary of a Report of 72314 Cases From the Chinese Center for Disease Control and Prevention. JAMA 2020.

5. Zhou F, Yu T, Du R, Fan G, Liu Y, Liu Z, Xiang J, Wang Y, Song B, Gu X, Guan L, Wei Y, Li H, Wu $\mathrm{X}$, Xu J, Tu S, Zhang Y, Chen H, Cao B. Clinical course and risk factors for mortality of adult inpatients with COVID-19 in Wuhan, China: a retrospective cohort study. The Lancet 2020.

6. Lee P, Hu Y, Chen P, Huang Y, Hsueh P. Are children less susceptible to COVID-19? Journal of Microbiology, Immunology and Infection 2020.

7. R Y, Y Z, Y L, L X, Y G, Q Z. Structural basis for the recognition of SARS-CoV-2 by full-length human ACE2. Science (New York, N.Y.) 2020;367(6485):1444-1448.

8. Cai J, Xu J, Lin D, Yang Z, Xu L, Qu Z, Zhang Y, Zhang H, Jia R, Liu P, Wang X, Ge Y, Xia A, Tian H, Chang H, Wang C, Li J, Wang J, Zeng M. A Case Series of children with 2019 novel coronavirus infection: clinical and epidemiological features. Clinical infectious diseases : an official publication of the Infectious Diseases Society of America 2020.

9. Zou L, Ruan F, Huang M, Liang L, Huang H, Hong Z, Yu J, Kang M, Song Y, Xia J, Guo Q, Song T, He J, Yen H, Peiris M, Wu J. SARS-CoV-2 Viral Load in Upper Respiratory Specimens of Infected Patients. NEW ENGL J MED 2020;382(12):1177-1179.

10. G L, M P. Laboratory abnormalities in patients with COVID-2019 infection. CLIN CHEM LAB MED 2020 .

11. Li W, Cui H, Li K, Fang Y, Li S. Chest computed tomography in children with COVID-19 respiratory infection. PEDIATR RADIOL 2020.

12. Xu X, Wu X, Jiang X, Xu K, Ying L, Ma C, Li S, Wang H, Zhang S, Gao H, Sheng J, Cai H, Qiu Y, Li L. Clinical findings in a group of patients infected with the 2019 novel coronavirus (SARS-Cov-2) outside of 
Wuhan, China: retrospective case series. BMJ 2020:m606.

13. Sun D, Li H, Lu X, Xiao H, Ren J, Zhang F, Liu Z. Clinical features of severe pediatric patients with coronavirus disease 2019 in Wuhan: a single center's observational study. WORLD J PEDIATR 2020.

14. Shi H, Han X, Jiang N, Cao Y, Alwalid O, Gu J, Fan Y, Zheng C. Radiological findings from 81 patients with COVID-19 pneumonia in Wuhan, China: a descriptive study. The Lancet Infectious Diseases 2020.

15. Xia W, Shao J, Guo Y, Peng X, Li Z, Hu D. Clinical and CT features in pediatric patients with COVID-19 infection: Different points from adults. PEDIATR PULM 2020.

16. Wu J, Li W, Shi X, Chen Z, Jiang B, Liu J, Wang D, Liu C, Meng Y, Cui L, Yu J, Cao H, Li L. Early antiviral treatment contributes to alleviate the severity and improve the prognosis of patients with novel coronavirus disease (COVID-19). J INTERN MED 2020.

17. Ludvigsson JF. Systematic review of COVID-19 in children shows milder cases and a better prognosis than adults. ACTA PAEDIATR 2020.

18. KL S, YH Y, RM J, TY W, DC Z, Y J, XX L, RM J, YJ Z, BP X, ZD X, ZS L, XW L, LK L, YX S, SN S, Y B, M L, G L, JK D, WJ L, LJ X, M L, YX C, LP Y, JF L, JB S, LW G, YY W, XF W. Updated diagnosis, treatment and prevention of COVID-19 in children: experts' consensus statement (condensed version of the second edition). World journal of pediatrics : WJP 2020.

\section{Hosted file}

Table 1.docx available at https://authorea.com/users/326905/articles/454611-clinicalfindings-and-treatments-of-21-children-with-covid-19-a-comparison-with-their-families

\section{Hosted file}

Table 2.docx available at https://authorea.com/users/326905/articles/454611-clinicalfindings-and-treatments-of-21-children-with-covid-19-a-comparison-with-their-families

\section{Hosted file}

Table 3.docx available at https://authorea.com/users/326905/articles/454611-clinicalfindings-and-treatments-of-21-children-with-covid-19-a-comparison-with-their-families

\section{Hosted file}

Table 4.docx available at https://authorea.com/users/326905/articles/454611-clinicalfindings-and-treatments-of-21-children-with-covid-19-a-comparison-with-their-families 